\title{
VALEUR ALIMENTAIRE DU TOURTEAU DE SOJA INFLUENCE DU TRAITEMENT THERMIQUE
}

PAR

\section{R. FEVRIER}

Station de Recherches sur l'Élevage, Centre National de Recherches

Zootechniques, Jouy-en-Josas.

\section{et A. GASNIER et J. P. VACHEL}

Institut Professionnel de Contrôle et de Recherches Scientifiques des Industries de l'Alimentation Animale, Paris.

\section{PLAN DU MÉMOIRE}

\section{I. - Mesures sur porcs. \\ II. - Mesures sur rats : \\ a) Digestibilités. \\ b) Courbes de croissance.}

\section{I. - Mesures SUR PORCS}

La matière grasse du soja peut être extraite de la graine par différents procédés. L'attention des praticiens, comme celle des chercheurs, a été attirée depuis longtemps par l'importance que présentait la nature de ce traitement pour la valeur alimentaire du tourteau.

De nombreux travaux ont montré qu'une cuisson en présence de vapeur d'eau améliorait l'efficacité du tourteau et prévenait même l'apparition de certains troubles consécutifs à l'ingestion de soja cru. JACQUoT et al. (2) ont publié récemment une très complète mise au point de la question.

En I949, une organisation professionnelle nous demanda de comparer la "valeur alimentaire " de trois tourteaux de soja provenant de trois usines utilisant des procédés de traitement différents.

Les renseignements suivants nous ont été donnés :

\section{Tourteau $n^{0} 1-$ Bordeaux.}

Après laminage, les graines de soja sont envoyées dans un extracteur continu Hansa-Murie, où elles sont traitées à l'essence B $60 / 80$. Les tourteaux déshuilés et encore chargés d'essence sont séchés dans des vis sécheuses chauf- 
fées à la vapeur. La température finale à laquelle est porté le tourteau dans les deux dernières vis sécheuses est de $130^{\circ}$; la durée du passage à cette température est de $I_{5}$ minutes.

L'opération se fait en présence de vapeur d'eau. L'humidité de la farine durant ce chauffage est voisine de 15 p. Ioo.

\section{Tourteau $n^{\mathrm{0}} 2-$ Marseille.}

Les graines de soja sont traitées dans des laminoirs spéciaux, en vue de leur préparation pour l'extraction. La marchandise est reprise et amenée dans des appareils fixes, où elle est traitée par solvant. Elle est ensuite portée à $\mathbf{2} 20^{\circ}$ par la vapeur d'eau, dans des appareils clos, en vue d'effectuer l'opération de cuisson. Pour terminer, le séchage a lieu dans un matériel à double enveloppe de vapeur, spécialement conçu pour éviter l'altération du produit.

\section{Tourteau $n^{0} 3-$ Paris.}

La fabrication comporte l'extraction par essence B 60/80 de la graine de soja convenablement broyée et laminée.

Après quelques heures de contact avec l'essence à la température d'environ 45 à $50^{\circ}$, l'essence d'imbibition du tourteau est chassée par un courant de vapeur. De ce fait, le tourteau se trouve porté à $105^{\circ}$ environ pendant 40 minutes, son humidité s'élevant autour de $20 \%$ Le tourteau est ensuite séché dans un séchoir continu, où il séjourne 20 minutes à la température de $80^{\circ}$.

Ce tourteau avait été broyé, dépelliculé et bluté sur tamis $7 \mathrm{XX}$.

Les trois tourteaux ont été examinés aux Laboratoires de Zootechnie de l'Institut National Agronomique et de l'Institut Professionnel de Contrôle et de Recherches Scientifiques des Industries de l'Alimentation Animale; les teneurs en acides aminés ont été calculées d'après les dosages effectués par le Laboratoire de Biochimie du Collège de France ( ${ }^{\mathbf{1}}$ ).

Voici leur composition (pour roo g de produit brut) :

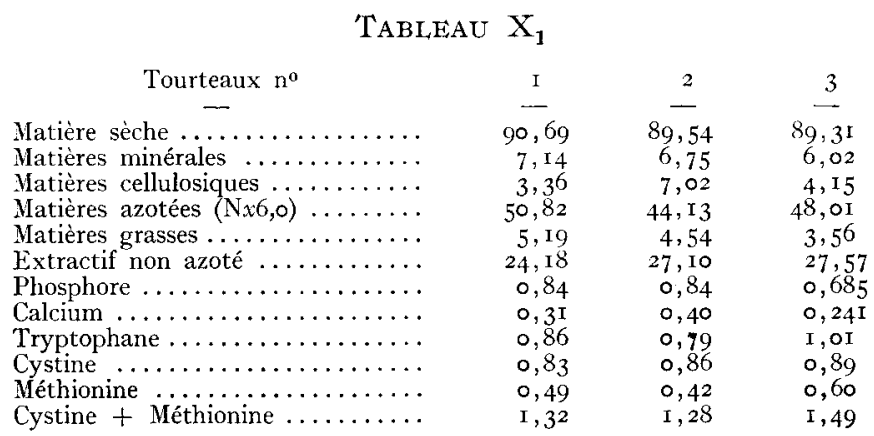

(1) Nous remercions le Professeur J. Roche d'avoir bien voulu faire procéder à ces dosages. 
Nous disposions à cette époque de I2 porcelets Large White répartis en trois groupes (I3-I4-I5). Leur poids moyen était alors :

$\begin{array}{ll}\text { groupe I3 } & 22 \mathrm{~kg} \\ \text { groupe I4 } & 28 \mathrm{~kg} \\ \text { groupe I5 } & 28 \mathrm{~kg}\end{array}$

Deux séries d'observations ont été réalisées sur ces animaux.

\section{EXPERIENCE I}

Le plan de rationnement fut le suivant :

\begin{tabular}{|c|c|}
\hline Période & Durée \\
\hline $\bar{I}$ & 2 semaines \\
\hline II & $2 "$ \\
\hline III & " \\
\hline IV & " \\
\hline $\mathrm{V}$ & 3 \\
\hline
\end{tabular}

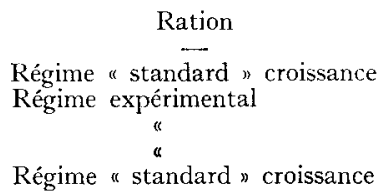

Les rations furent composées de façon à présenter des caractéristiques voisines : taux d'azote et de matières cellulosiques, en particulier :

Régime "standard " croissance.

Orge ................. 73

Levure de distillerie ........ 5

Farine de viande $\ldots \ldots \ldots \ldots .6$

Farine de poisson .............

Tourteau d'arachide ...........

Mélange minéral
Régime expérimental.

Mélange minéral ....... 3

Orge ................. 78

Farine de viande $\ldots \ldots \ldots$. 4

Tourteau de soja $\ldots \ldots \ldots$ I. I5

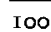

La nature du tourteau était choisie de façon à ce que chacun des trois groupes reçoive successivement les trois types de tourteau au cours des 3 périodes II-III-IV et que, pendant chaque période, chacun des 3 tourteaux soit distribué à l'un des 3 groupes.

Le dispositif suivant fut utilisé :

$\begin{array}{lrrr} & \text { Période II } & \text { Période III } & \text { Période IV } \\ \text { Groupe I3 } & \text { tourt. } & - & - \\ \text { Groupe 1 } & 2 & 2 & 3 \\ \text { Groupe I5 } & 3 & 3 & \text { I } \\ \text { I5 } & & \text { I } & 2\end{array}$

Les animaux étaient alimentés trois fois par jour et rationnés d'après leur appétit, comme nous l'avons décrit par ailleurs (Ann. Agron. no 4, I947) (3-4).

Voici les résultats obtenus, en ce qui concerne le gain de poids (G), la consommation (C) et l'indice de consommation (I) - en $\mathrm{kg}$ :

\begin{tabular}{|c|c|c|c|c|c|c|c|c|c|c|c|c|c|c|c|}
\hline \multirow{2}{*}{ 总 } & \multicolumn{3}{|c|}{ Période I } & \multicolumn{3}{|c|}{ Période II } & \multicolumn{3}{|c|}{ Période III } & \multicolumn{3}{|c|}{ Période IV } & \multicolumn{3}{|c|}{ Période V } \\
\hline & G & $\mathrm{C}$ & I & G & C & I & G & C & I & G & C & I & G & $\mathrm{C}$ & I \\
\hline 13 & & 69 & 2,87 & 24 & 83 & 3,46 & I3 & 89.5 & 6,88 & 34 & 109,5 & 3,22 & 34 & I 28 & 3,76 \\
\hline I4 & 24 & 83 & 3,46 & 24 & 96,5 & 4,02 & 22 & 103 & 4,68 & I7 & 104 & $6, \mathrm{II}$ & $3^{6}$ & II 8 & 3,27 \\
\hline 15 & $2 \mathrm{I}$ & 76,5 & 3,64 & 23 & 83 & 3,60 & 8 & 89,5 & $\mathrm{rI}, \mathrm{IB}$ & 34 & 109,5 & 3,22 & 37 & $\mathrm{I} 2 \mathrm{I}$ & 3,27 \\
\hline
\end{tabular}


Rapportés à chaque groupe de porcs, les résultats pour l'ensemble des 5 périodes sont les suivants (en $\mathrm{kg}$ ) :

\begin{tabular}{|c|c|c|c|c|}
\hline & Gain total & $\begin{array}{l}\text { Gain moyen } \\
\text { quotidien } \\
-\end{array}$ & Consommation & $\begin{array}{c}\text { Indice de } \\
\text { consommation }\end{array}$ \\
\hline Groupe ${ }_{13}$ & I 29 & 0,419 & 479 & $3,7 \mathrm{I}$ \\
\hline Groupe I4 & 123 & 0,399 & 504,5 & 4,10 \\
\hline Groupe 15 & I 23 & 0,399 & 479,5 & 3,90 \\
\hline Tourleaux & 3 & EIIA & & \\
\hline & no1 & $n^{\circ} 2$ & $n \circ 3$ & \\
\hline
\end{tabular}

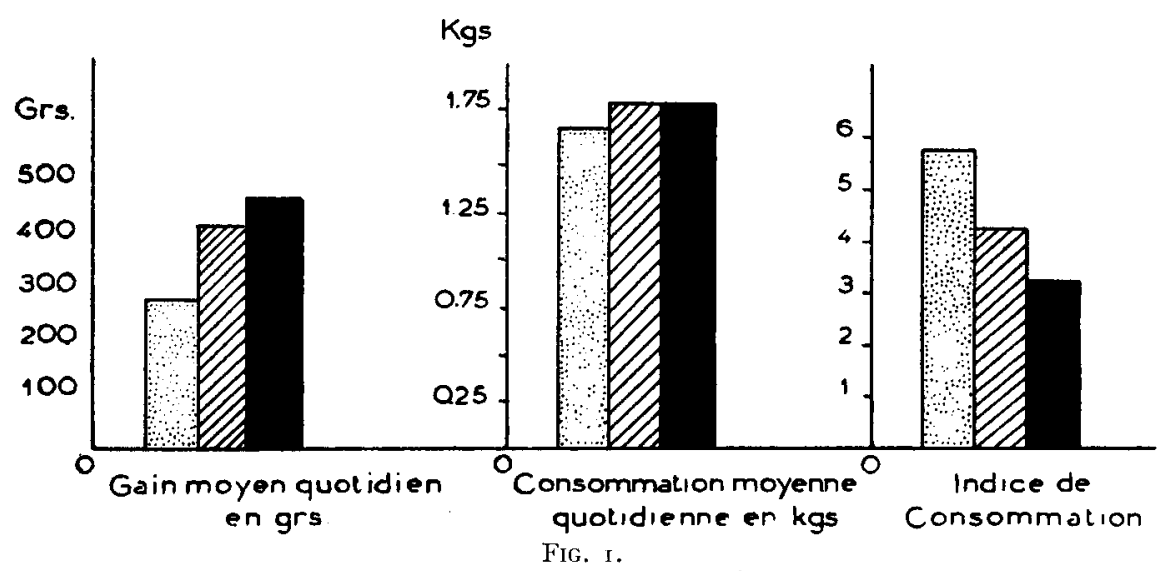

Rapportés atxx porcs ayant reçu la même ration expérimentale au cours des 3 périodes II-III-IV, les résultats sont exprimés par le tableau suivant (en $\mathrm{kg}$ ) :

\begin{tabular}{|c|c|c|c|c|c|c|c|c|c|c|c|c|c|c|}
\hline \multirow{2}{*}{$\begin{array}{c}\text { Animaux } \\
\text { recevant } \\
\text { tourt. }\end{array}$} & \multicolumn{3}{|c|}{ Période II } & \multicolumn{3}{|c|}{ Période III } & \multicolumn{3}{|c|}{ Période IV } & \multicolumn{5}{|c|}{ Total } \\
\hline & G & C & I & G & C & I & G & C & I & $\begin{array}{c}G \\
\text { tot. }\end{array}$ & $\begin{array}{c}\mathrm{G} \\
\text { quot. }\end{array}$ & $\begin{array}{c}\mathrm{C} \\
\text { tot. }\end{array}$ & $\begin{array}{c}\mathrm{C} \\
\text { quot. }\end{array}$ & I \\
\hline I & 24 & 83 & $3,4^{6}$ & 8 & 89,5 & I I, 18 & 17 & & $6, \mathrm{II}$ & 49 & 0,292 & 276,5 & $x, 65$ & 5,64 \\
\hline 2 & 24 & $9^{6,5}$ & & 13 & 89,5 & $\begin{array}{r}6,88 \\
\end{array}$ & 34 & 109,5 & 3,22 & 71 & $0,4^{23}$ & 295,5 & $\begin{array}{r}1,76 \\
-6\end{array}$ & 4,16 \\
\hline 3 & 23 & 83 & 3,60 & 22 & 103 & 4,68 & 34 & 109,5 & 3,22 & 79 & $0,47^{\circ}$ & 295,5 & $\mathrm{I}, 76$ & 3,74 \\
\hline
\end{tabular}

Ils peuvent également être présentés sous la forme suivante :

$\begin{array}{ccccc}\text { Tourteau } & \begin{array}{c}\text { Poids moyen } \\ \text { des animaux }\end{array} & \begin{array}{c}\text { Gain moyen } \\ \text { quotidien }\end{array} & \begin{array}{c}\text { Consommation } \\ \text { moyenne } \\ \text { quotidienne }\end{array} & \begin{array}{c}\text { Indice de } \\ \text { consommation }\end{array} \\ - & - & - & - & - \\ \mathbf{I} & 39,3 & 0,292 & \mathrm{~kg} & - \\ 2 & 39,2 & 0,423 & 1,65 & 5,64 \\ 3 & 39,9 & 0,470 & 1,76 & 4,16 \\ & & 1,76 & 3,74\end{array}$

Nous remarquons ainsi que l'appétence de la ration I semble légèrement inférieure à celle des deux autres. Peut-on expliquer ainsi la médiocrité de la croissance observée sur le lot I ? 
En appliquant la méthode décrite par ailleurs (I) pour compare rles efficacités, et en prenant pour unité l'efficacité du lot $I$, nous avons :

\begin{tabular}{cc} 
Lot & Efficacité \\
\hline I & I \\
2 & I , I6 \\
3 & I , 26
\end{tabular}

Ainsi, la ration I se révèle non seulement moins appétente, mais encore moins efficace que la ration 2. Ceci explique l'indice de consommation élevé que nous avons constaté.

D'autre part, la ration du lot 3 , d'une appétence égale à celle du lot 2 , est plus efficace.

Ces différences tiennent-elles au traitement thermique, à la nature des graines ou à leur état de conservation? Il ne nous est pas possible d'y répondre ici. Mais notons que leur richesse différente en acides aminés, et notamment en tryptophane pourrait rendre compte de cas observations.

\section{EXPERIENCE II}

Nous avons décidé de répéter les essais. Cependant, les porcs devenant plus âgés et par là, moins sensibles à d'éventuelles carences protéiques, nous avons supprimé la farine de viande de la ration pour sensibiliser, en quelque sorte, les animaux à un déséquilibre éventuel de la ration. Le protocole employé fut le même : après une période intermédiaire de 3 semaines séparant les deux expériences, nous avons observé les effets des 3 tourteaux au cours de 3 périodes expérimentales de deux semaines. Le plan de rationnement fut le suivant :

\begin{tabular}{|c|c|c|c|c|}
\hline & $\begin{array}{c}\text { Période } \\
\text { intermédiaire. }\end{array}$ & $\begin{array}{c}\text { Période I } \\
2 \text { semaines. }\end{array}$ & $\begin{array}{l}\text { Période II } \\
2 \text { semaines. }\end{array}$ & $\begin{array}{l}\text { Période III } \\
2 \text { semaines. }\end{array}$ \\
\hline e $I_{3}$ & al. standard & rat. I & 2 & 3 \\
\hline & - & 2 & 3 & I \\
\hline Groupe i5 & $\ldots$ & 3 & I & 2 \\
\hline
\end{tabular}

La formule des rations expérimentales fut:

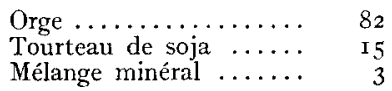

Les résultats obtenus figurent dans les tableaux suivants :

a) Période intermédiaire (3 semaines)

\begin{tabular}{lccc} 
& Gain & Consommation & Indice \\
\cline { 2 - 3 } & - & - & - \\
Groupe 13 & 34 & $\mathrm{~kg}$ & $\mathrm{~kg}$ \\
Groupe I4 & 36 & II8 & 3,74 \\
Groupe 15 & 37 & I21 & 3,28 \\
15 & & 3,43
\end{tabular}


b) Tableau par groupe (en $\mathrm{kg}$ )

\begin{tabular}{|c|c|c|c|c|c|c|c|c|c|c|c|c|}
\hline \multirow{2}{*}{ 岂 } & \multicolumn{3}{|c|}{ Période I } & \multicolumn{3}{|c|}{ Période II } & \multicolumn{3}{|c|}{ Période III } & \multicolumn{3}{|c|}{ Total général } \\
\hline & G & C & I & G & C & I & G & C & I & G & C & I \\
\hline 13 & 28 & 109,5 & $3,9 \mathrm{I}$ & 39 & I 43 & 3,67 & 39 & 162,5 & 4,17 & 106 & 4I 5 & 3,91 \\
\hline 14 & 35 & I 35,5 & 3,87 & 46 & 153,5 & 3.34 & 35 & $I_{53}$ & 4.37 & 116 & $44^{2}$ & $3,8 \mathrm{I}$ \\
\hline I 5 & $3^{8}$ & I 38,5 & 3,64 & $3^{2}$ & I $4 \mathrm{I}$ & 4,06 & 42 & 164 & 3,92 & I I 2 & 443,5 & 3,96 \\
\hline
\end{tabular}

c) Tableau par lot (en $\mathrm{kg}$ )

\begin{tabular}{|c|c|c|c|c|c|c|}
\hline \multirow[b]{2}{*}{ Lot I } & Période I & Période II & Période III & \multicolumn{3}{|c|}{ Total général } \\
\hline & I09,5 & I $4 \mathrm{I}$ & I 53 & 95 & 403,5 & 4,24 \\
\hline Lot 2 & $\mathrm{r} 35,5$ & $\mathrm{I} 43$ & 164 & 116 & $44^{2}, 5$ & $3,8 \mathrm{r}$ \\
\hline Lot 3 & I 38,5 & I 53,5 & 162,5 & I 23 & 454,5 & 3,69 \\
\hline
\end{tabular}

Ils peuvent être présentés de la façon suivante :

\begin{tabular}{|c|c|c|c|c|}
\hline & Poids moyen & $\begin{array}{c}\text { Gain moyen } \\
\text { quotidien }\end{array}$ & $\begin{array}{l}\text { Consommation } \\
\text { moyenne } \\
\text { quotidienne } \\
-\end{array}$ & $\begin{array}{l}\text { Indice de } \\
\text { consommation } \\
-\end{array}$ \\
\hline Lot 1 & $7 \mathrm{r}, 2 \mathrm{~kg}$ & $0,565 \mathrm{~kg}$ & 2,40 & 4,24 \\
\hline Lot 2 & $70,25 \|$ & 0,690 & 2,63 & $3,8 \mathrm{I}$ \\
\hline Lot 3 & 70,75 & $0,73^{2}$ & 2,70 & 3,69 \\
\hline
\end{tabular}

Les résultats que nous avions observés dans la première expérience se trouvent donc confirmés, avec les corrections qu'ont pu y ajouter les variations d'âge des animaux, à savoir :

- plus grande sensibilité aux différences d'appétence, comme déjà plusieurs observations antérieures nous l'ont suggéré,

- sensibilité moindre à la qualité de l'azote de la ration.

Le calcul montre, en effet, que l'efficacité des trois rations est identique et que les différences observées dans l'indice proviennent de l'appétence qui, modifiant le volume de l'ingestion, agit sur la vitesse de croissance.

\section{III. - NOUVELLE EXPERIENCE}

La cause de ces différences ne pouvant être mise en évidence, nous avons poursuivi nos investigations.

Nous avons demandé à la Société qui nous avait déjà livré le tourteau soja $n^{\circ} \mathrm{I}$, de nous fournir deux tourteaux obtenus à partir d'un même lot de de graines:

- le tourteau $\mathrm{n}^{0} 4$, qui aurait été traité comme le $\mathrm{n}^{\mathrm{O}} \mathrm{I}$,

- le tourteau $n^{\circ} 5$, qui aurait été soumis à une cuisson plus forte. 
Les analyses, effectuées par les mêmes laboratoires que précédemment, ont donné les résultats suivants (pour roo $g$ de produit brut):

\begin{tabular}{|c|c|c|}
\hline \multicolumn{3}{|l|}{ TABLEAU $\mathrm{X}_{2}$} \\
\hline & 4 & 5 \\
\hline Matière sèche... & 9 I, 26 & 90,45 \\
\hline Matières minérales .. & 6,94 & 6,44 \\
\hline Matières cellulosiques ... & 3,02 & 3,69 \\
\hline Matières azotées $(N \times 6,0)$ & 48,26 & 46,60 \\
\hline Matières grasses ............ & 3,83 & 3,12 \\
\hline Extractif non azoté........ & $29,2 \mathrm{I}$ & 30,60 \\
\hline Phosphore ............. & $0,9 \mathrm{I}$ & 0,90 \\
\hline Calcium ........ & 0,35 & 0,23 \\
\hline Tryptophane & $\mathrm{I}, \mathrm{II}$ & $\mathrm{I}, 05$ \\
\hline Cystine ..... & 0,65 & 0,63 \\
\hline Méthionine $\ldots \ldots, \ldots \ldots \ldots$ & 0,53 & 0,47 \\
\hline Cystine + Méthionine ... & 1,18 & $\mathrm{I}, \mathrm{IO}$ \\
\hline
\end{tabular}

Nous disposions alors de 36 porcelets, en 9 groupes de 4 . Après 5 semaines d'observation, nous les avons classés en trois lots de I2 qui avaient donné les résultats suivants avec l'aliment standard (en $\mathrm{kg}$ ).

\begin{tabular}{|c|c|c|c|c|c|}
\hline & \multicolumn{2}{|c|}{ Poids moyen } & \multicolumn{3}{|c|}{ Gain moyen $\begin{array}{c}\text { Consommation } \\
\text { moyenne }\end{array} \stackrel{\text { Indice moyen }}{\mathrm{de}}$} \\
\hline & début & fin & quotidien & quotidienne & consommation \\
\hline Lot I & 30,9 & $5 \mathrm{I}, 25$ & 0,580 & $\mathrm{I}, 83$ & $3, \mathrm{I} 4$ \\
\hline ot 4 & 30,3 & $5^{1,}, 17$ & 0,595 & I, 87 & $3, \mathrm{I} 4$ \\
\hline ot 5 & 30,9 & 51,58 & 0,590 & I, 87 & 3 , IO \\
\hline
\end{tabular}

Nous avons alimenté ces animaux avec une ration composée d'orge 82 $\mathrm{p}$, Ioo, de tourteau de soja I $5 \mathrm{p}$. Ioo et de mélange minéral $3 \mathrm{p}$. Ioo. Le lot I reçut le tourteau de soja $n^{\circ}$, le lot 4 le tourteau $\mathbf{n}^{0} 4$ et le lot 5 le tourteau $n^{\circ} 5$.

L'analyse des rations ainsi constituées donna les résultats suivants p. 1000 :

\begin{tabular}{|c|c|c|c|c|c|c|}
\hline & $\begin{array}{l}\text { Mat. } \\
\text { sèche } \\
-\end{array}$ & $\begin{array}{c}\text { Wat. } \\
\text { azotées } \\
\text { - }\end{array}$ & $\begin{array}{l}\text { Wat. } \\
\text { grasses } \\
-\end{array}$ & $\begin{array}{l}\text { Mat. } \\
\text { cellul. } \\
\text { - }\end{array}$ & $\begin{array}{l}\text { Mat. } \\
\text { minérales } \\
\text { _ }\end{array}$ & Acidité \\
\hline Ration $n^{0}$ I & 894 & I 64 & 3I & 75 & 50 & $\mathrm{~J}, 6$ \\
\hline Ration $n^{\circ} 4$ & 915 & 162 & 30 & $8 \mathrm{I}$ & 45 & $\mathrm{I}, 5$ \\
\hline Ration $n^{\circ} 5$ & 910 & I60 & 29 & 83 & $5^{2}$ & 1,7 \\
\hline
\end{tabular}

Ainsi, les animaux recevaient des rations isoazotées, composées des mêmes produits, le seul élément de variation étant la nature du tourteau de soja utilisé :

- les rations I et 4 contenaient des tourteaux fabriqués dans les mêmes conditions, mais provenant de graines différentes,

- les rations 4 et 5 contenaient des tourteaux fabriqués à partir des mêmes graines, mais avec des procédés différents.

Ainsi espérions-nous, d'après le comportement des animaux, distinguer l'influence de ces deux facteurs sur la "valeur alimentaire " du tourteau de soja. 
Les résultats portant sur 5 semaines d'observation sont rapportés dans le tableau suivant (en $\mathrm{kg}$ ) :

\begin{tabular}{|c|c|c|c|c|c|}
\hline & \multicolumn{2}{|c|}{ Poids moyen } & \multirow{2}{*}{$\begin{array}{c}\text { Gain } \\
\text { moyen } \\
\text { quotidien }\end{array}$} & \multirow{2}{*}{$\begin{array}{c}\text { Consommation } \\
\text { moyenne } \\
\text { quotidienne }\end{array}$} & \multirow[b]{2}{*}{$\begin{array}{l}\text { Indice de } \\
\text { consommation }\end{array}$} \\
\hline & début & fin & & & \\
\hline t $I$ & $5^{\mathrm{I}, 2}$ & 66,5 & $\circ, 435$ & 2,06 & 4,74 \\
\hline Lot 4 & $5 \mathrm{I}, 2$ & 63,8 & 0,357 & 2,00 & 5,60 \\
\hline Lot 5 & $5 \mathrm{I}, 6$ & 68,7 & 0,488 & $2,5^{8}$ & 4,32 \\
\hline
\end{tabular}

Si nous comparons deux à deux les résultats obtenus, nous constatons que :

I $^{0}$ les 2 tourteaux ayant subi même traitement thermique $(I-4)$ présentent une différence concernant:

- la vitesse de croissance $(435-357 \mathrm{~g})$,

- l'indice de consommation $(4,74-5,60)$ et, par contre, une similitude dans la consommation $(2,06-2,00)$.

Ces observations mettent en évidence la supériorité du tourteau I. Par la méthode employée précédemment, nous pouvons calculer le rapport des efficacités : en prenant pour unité l'efficacité du lot I, celle du lot 4 est de $0,86-$ donc la différence est sensible.

Il semble ainsi établi que l'origine de la graine a, ici une influence sur l'efficacité du tourteau, mais non sur son appétence.

$2^{\circ}$ les deux tourteaux provenant du même lot de graines (4-5) accusent également des différences pour :

- la vitesse de croissance $(357-488)$,

- 1'indice de consommation $(5,60-4,32)$,

- la consommation $(2,00-2,58)$.

Le calcul montre qu'en prenant l'efficacité de la ration I comme unité, l'efficacité de la ration 5 est de $\mathrm{I}, 05$. Le tourteau $\mathrm{n}^{\circ} 5$ présente donc, sur le tourteau 4 , un net avantage provenant de son appétence supérieure et probablement, d'une meilleure efficacité. La cuisson plus forte qu'il a subie a donc sensiblement amélioré sa valeur alimentaire globale, puisque l'indice de consommation des animaux du lot 5 est inférieur de 30 p. Ioo à celui des animaux du lot 4 .

Pour les trois échantillons examinés, les différences provenant du traitement thermique portent sur l'appétence et, à un degré moindre, sur l'efficacité, alors que les différences provenant de l'origine des graines ne portent que sur l'efficacité. Il serait toutefois présomptueux de vouloir généraliser cette observation.

\section{CONCLUSION}

I. - Les trois expériences que nous avons effectuées nous ont permis d'étudier la "valeur alimentaire " de 5 tourteaux de soja, différents les uns des autres par l'origine de la matière première et par le traitement thermique subi à l'huilerie. 
II. - Ces tourteaux présentaient d'assez notables différences dans leur appétence et dans leur efficacité, provenant à la fois du traitement industriel et de la qualité de la graine utilisée. Il importe donc, pour des études ultérieures, de veiller à cette double cause de variation.

III. - Nos observations confirment les résultats des travaux antérieurs qui avaient mis en évidence l'amélioration considérable que provoque, sur la valeur alimentaire du tourteau de soja, la cuisson en présence de vapeur d'eau.

IV. - Il semble, d'après les échantillons étudiés, que l'influence de la graine se limite à l'efficacité du tourteau, alors que le traitement thermique agit nettement sur son appétence et, vraisemblablement, sur son efficacité.

\section{II. - MESLRES SUR RITS}

I1 pourrait être intéressant de comparer la valeur alimentaire de tourteaux de soja destinés aux animaux de ferme en utilisant le rat comme animalréactif : les mesures sont de réalisation plus facile et le matériel nécessaire beaucoup moins couteux.

Nous avons donc entrepris deux séries de recherches sur les mêmes tourteaux de soja qui ont été utilisés dans les expériences précédemment rapportées. L'une des séries de mesures a été réalisée sur des rats mâles adultes; elle a permis de déterminer la digestibilité des rations employées et des différents constituants de ces rations, ainsi que d'établir le bilan azoté des animaux. L'autre série a été réalisée sur de jeunes rats des deux sexes pris au sevrage, afin d'observer la croissance des animaux; les résultats de cette $2^{\mathbf{e}}$ série sont ainsi plus directement comparables à ceux qui ont été obtenus sur porcs.

\section{A. - Etude des digestibilités}

\section{a) Protocole expérimental}

5 séries d'expériences ont été effectuées avec les tourteaux de soja à étudier et une $6^{\mathrm{e}}$ série, servant de témoin, avec un aliment complet (AEC) pour rats. Ces différents aliments furent mélangés en proportions variables avec de l'orge et complémentés par un composé minéral. L'orge et le composé minéral étaient les mêmes que ceux qui avaient été employés dans les expériences de croissance sur porcs.

Les rations ont été établies de façon à obtenir une teneur en matières azotées voisine de $2 \mathrm{I}-24 \mathrm{p}$. Ioo pour le régime brut. Le tableau I indique la composition des aliments d'expérience autres que les tourteaux de soja dont la composition a été donnée plus haut.

Dans la suite de l'exposé, nous désignerons les rations par les numéros des tourteaux de soja entrant dans leurs compositions respectives. 
Tableau I

Composition des aliments d'expérience autres que les tourteaux de soja

(g pour roo $\mathrm{g}$ brut)

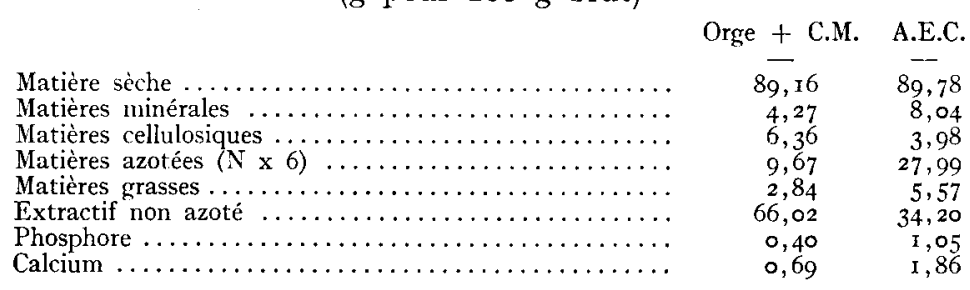

Voici le schéma des expériences :

\begin{tabular}{|c|c|c|c|c|c|}
\hline $\begin{array}{c}\text { No de la } \\
\text { série }\end{array}$ & $\begin{array}{l}\text { No du } \\
\text { soja }\end{array}$ & $\begin{array}{c}\text { Durée } \\
\text { d'expérience }\end{array}$ & de soja & $\begin{array}{l}\% \\
\text { d'orge }\end{array}$ & $\begin{array}{l}\text { Nombre } \\
\text { de rats }\end{array}$ \\
\hline- & - & - & - & - & - \\
\hline$\frac{1}{V}$ & $\mathrm{r}$ & II j. & 30 & 70 & 5 \\
\hline V & 2 & I8 j. & 35 & 65 & 5 \\
\hline II & 3 & I I $\bar{j}$. & 30 & 70 & 5 \\
\hline III & 4 & ro j. & 30 & 70 & 5 \\
\hline IV & 5 & Io $\mathrm{j}$. & 35 & 65 & 5 \\
\hline VI & $A E C$ & I $2 \mathrm{j}$. & $65 \%$ AEC & 35 & 4 \\
\hline
\end{tabular}

Chaque série d'expérience est précédée d'une période pré-expérimentale d'adaptation au régime, variant de 7 à ro jours.

Chaque jour, on prélève dans la pâtée préparée pour les animaux, un échantillon d'environ $50 \mathrm{~g}$. A la fin de l'expérience, le mélange des $n$ échantillons qui ont été séchés est homogénéisé et analysé, et les résultats de l'analyse de l'échantillon global sont comparés avec les résultats théoriques obtenus par calcul à partir de la composition des matières premières. Les deux séries de valeurs concordent en général d'une façon très satisfaisante, avec une précision en rapport avec celle des manipulations et des pesées. L'analyse de ces échantillons caractéristiques de chaque série figure dans le tableau no II :

TABLEAU II

Composition des rations

(g pour Ioo $\mathrm{g} \mathrm{sec}$ )

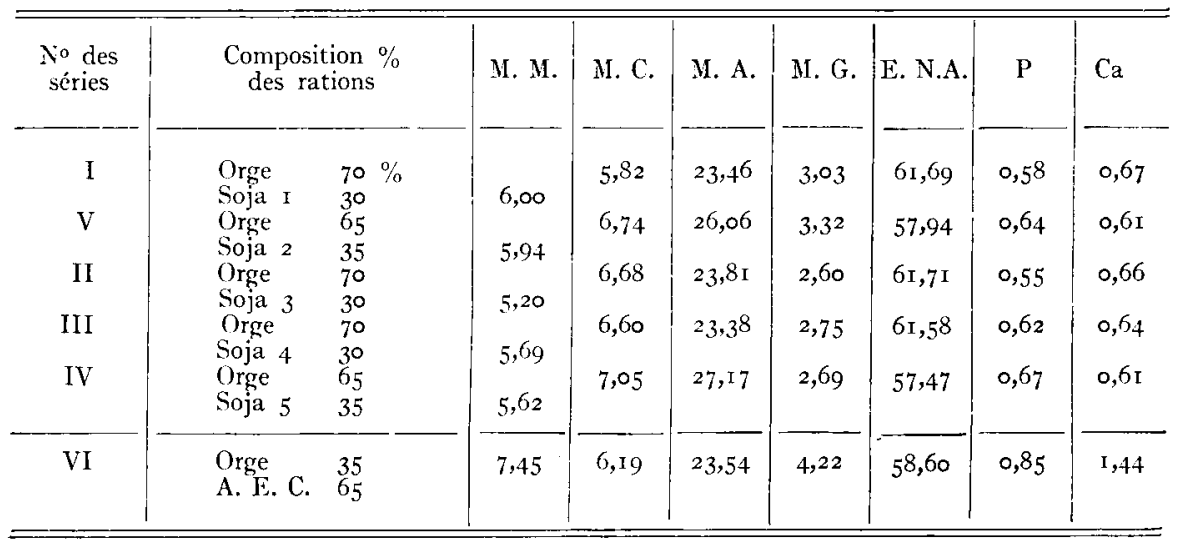




\section{b) Résultats}

Les digestibilités moyennes des différents éléments de la ration sont indiquées dans le tableau $n^{\circ}$ III :

\section{Tableau III}

Digestibilités brutes moyennes et erreurs standards pour les différents composants de la ration

\begin{tabular}{|c|c|c|c|c|c|c|}
\hline \multirow{2}{*}{ Éléments nutritifs } & \multicolumn{6}{|c|}{ No des sojas } \\
\hline & I & 2 & 3 & 4 & 5 & A. E. C. \\
\hline Matière sèche $\left(^{1}\right) \ldots \ldots \ldots \ldots \ldots \ldots$ & $\begin{array}{r}73,0 \\
-70,7\end{array}$ & $\begin{array}{r}72,7 \\
+0,6\end{array}$ & $\begin{array}{r}74,0 \\
+0,6\end{array}$ & $\begin{array}{r}73,2 \\
+\quad 0,7\end{array}$ & $\begin{array}{r}74,4 \\
\pm 0,6\end{array}$ & $\begin{array}{r}74,1 \\
+0,5\end{array}$ \\
\hline Matière organique $\ldots \ldots \ldots$ & $\begin{array}{r}74,9 \\
+\quad 18\end{array}$ & $\begin{array}{r}74,5 \\
+\quad 1,6\end{array}$ & $\begin{array}{r}75,9 \\
+\quad 1,7\end{array}$ & $\begin{array}{r}75,2 \\
+\quad 1,8\end{array}$ & $\begin{array}{r}76,4 \\
+\quad I, 7\end{array}$ & $\begin{array}{r}77,2 \\
+\quad 1,9\end{array}$ \\
\hline Matières minérales $\ldots \ldots \ldots \ldots \ldots$ & 44,5 & 46,6 & 43,2 & 42,7 & 45,3 & 28,8 \\
\hline Matières cellulosiques ... & $\begin{array}{r}+I, 4 \\
I, 3\end{array}$ & $\begin{array}{r}0,7 \\
9,6\end{array}$ & $\begin{array}{r}2,2 \\
9,7\end{array}$ & $\begin{array}{r} \pm 0,7 \\
20,6\end{array}$ & $\begin{array}{r} \pm, 6 \\
2 I, 2\end{array}$ & $\begin{array}{r} \pm 1,7 \\
30,5\end{array}$ \\
\hline Matières azotées. & $\begin{array}{r}+3,0 \\
73,8\end{array}$ & $\begin{array}{r}-4,9 \\
73,4\end{array}$ & $\begin{array}{r} \pm 7,2 \\
79,2\end{array}$ & $\begin{array}{r} \pm 4,4 \\
7 \mathrm{I}, 8\end{array}$ & $\begin{array}{r}+4,2 \\
78,0\end{array}$ & $\begin{array}{r} \pm 5,6 \\
75,8\end{array}$ \\
\hline & $\pm 1,8$ & $\pm 1,9$ & $\pm 1,2$ & $\pm 2,3$ & $\pm I, 3$ & $\pm 2,2$ \\
\hline Matières grasses $\left({ }^{2}\right) \ldots$ & - & $\begin{array}{r}56,0 \\
-+20\end{array}$ & $\begin{array}{r}48,7 \\
+\quad 0,7\end{array}$ & $\begin{array}{r}50,8 \\
+\quad 1,7\end{array}$ & $\begin{array}{r}59,3 \\
-+2,2\end{array}$ & $\begin{array}{r}60,8 \\
-\quad 18\end{array}$ \\
\hline Extractif non azoté ........ & - & 84,0 & 82,6 & 83,4 & 83,4 & 84,9 \\
\hline Phosphore . & 40,0 & $\begin{array}{r} \pm \mathrm{I}, 3 \\
37,6\end{array}$ & $\begin{array}{r} \pm 1,3 \\
28,7\end{array}$ & $\begin{array}{r} - \pm 1,6 \\
47,8\end{array}$ & $\begin{array}{r} \pm 2,5 \\
44,4\end{array}$ & $\begin{array}{r} \pm 0,8 \\
53,2\end{array}$ \\
\hline Calcium $\ldots$. & négatif & $\begin{array}{c} \pm 2,9 \\
\text { négatif }\end{array}$ & $\begin{array}{r} \pm, 5 \\
8,7 \\
\pm \quad 2, \mathrm{I}\end{array}$ & $\begin{array}{r} \pm 1,2 \\
4,6 \\
+\quad 1,0\end{array}$ & $\begin{array}{l} \pm 3,0 \\
\text { négatif }\end{array}$ & $\begin{array}{l} \pm 0,7 \\
\text { négatif }\end{array}$ \\
\hline
\end{tabular}

(1) Ces digestibilités ont été calculées à partir des ingesta et excreta totaux des 5 rats, obtenus pendant les i I jours d'expérience, alors que les autres digestibilités ne sont que les moyennes arithmétiques des 5 digestibilités individuelles obtenues pour chaque rat. Aussi les erreurs standards pour la matière sèche sont-elles nettement plus faibles, puisqu'elles sont calculées à l'aide de $5 n$ digestibilités au lieu de 5 comme pour les autres composants de la ration.

$\left({ }^{2}\right) C f$. page I 2. Matières grasses.

On remarque que les digestibilités moyennes de la matière sèche, de la matière organique et des matières minérales ne varient pratiquement pas lorsqu'on utilise successivement les différents sojas. Nous avons toutefois observé des différences individuelles sensibles, notamment en ce qui concerne la digestibilité de la matière sèche et celle de la matière organique : 2 de nos animaux sont nettement plus mauvais utilisateurs que leurs congénères.

Exemple: Digestibilité de la matière organique, rations 2 et 4 .

$\begin{array}{cccccc}\text { Rats no } & \text { I } & 2 & 3 & 5 & 6 \\ \text { Digest. ration 2 } & 73,9 & 78,5 & 77,8 & 72,1 & 70,3 \\ \text { Digest. ration 4 } & 75,0 & 80,0 & 78,1 & 72,6 & 70,2\end{array}$

Ces différences n'existent pas pour les matières minérales. Par ailleurs, on note que la digestibilité des matières minérales totales de la ration contenant l'aliment commercial (série VI) est significativement plus faible que celles que 
nous avons déterminées avec les rations contenant les sojas, rations cependant beaucoup moins bien équilibrées.

Matières cellulosiques: On pourrait penser, à la lecture du tableau III, que la digestibilité des matières cellulosiques des rations 4 et 5 est nettement supérieure à celle des rations I, 2 et 3 . Il n'en est rien. Les digestibilités moyenne des rations 4 et 5 (20p. Ioo environ) présentent des différences non significatives $(t=\mathrm{I}, 4$; signification pour 2,78$)$ avec celles des trois autres régimes dont la digestibilité est voisine de Io p. Ioo Ce résultat est dû à la très grande dispersion de valeurs individuelles dépendant de facteurs physiologiques incontrôlables : chacun sait que le rat est un très mauvais utilisateur des matières cellulosiques. On pourra remarquer la digestibilité relativement élevée de l'aliment commercial, due peut-être au meilleur équilibre de la ration. Enfin, signalons que les différences individuelles apparaissent ici encore, un des rats se révélant meilleur utilisateur de cellulose que les autres.

Exemple : Rations 2 et 5 .

\begin{tabular}{|c|c|c|c|c|c|}
\hline Rats $n^{\circ}$ & I & 2 & 3 & 5 & 6 \\
\hline Digest. ration 2 & $\overline{\mathrm{II}, \mathrm{I}}$ & $\overline{2 \mathrm{I}, 6}$ & $\overline{18,8}$ & $-\overline{3}, 2$ & $\overline{0,02}$ \\
\hline Digest. ration 5 & 15,0 & 33,4 & 28,8 & 16,5 & I 2,1 \\
\hline
\end{tabular}

Matières azotées totales: Nos résultats 'se classent en deux groupes distincts ; les rations $I, 2$ et 4 ont une digestibilité apparente de l'azote nettement plus faible que les régimes 3 et $5: 7$ I, 8 à 73,8 p. Ioo contre $78-79,2 \mathrm{p}$. IOO. Et si l'on prend comme référence la digestibilité des matières azotées totales du régime $\mathbf{n}^{0} 4: 7 \mathrm{r}, 8 \mathrm{p}$. Ioo, on peut montrer par le calcul que la différence observée est significative entre les rations 4 et $3(t=2,85)$ et presque significative entre cette même ration 4 et le régime $5(t=2,3$; signification pour $t=2,78)$.

Il est donc possible de mettre en évidence, chez le rat adulte, des variations d'utilisation digestive de l'azote fourni par des tourteaux de soja de même origine, ayant subi des traitements thermiques différents.

Matières grasses: Les dosages des matières grasses dans les fèces ont fait l'objet de nombreuses recherches dont les résultats n'étaient pas encore au point quand nous avons expérimenté le tourteau $n^{\circ}$ I. Aussi, les digestibilités des matières grasses et, par suite, celles de l'extractif non azoté, ne sont-elles pas indiquées pour l'expérience $n^{\circ}$ I. Les différences de digestibilité des rations 3 et 4 d'une part et 5 d'autre part, sont significatives (soja 3 et $5 ; t=4,8 \mathrm{I}$ soja 4 et $5, t=3,0$ ). Il est intéressant de noter que la ration 3 qui présentait la plus forte utilisation digestive des matières azotées est justement celle dont la digestibilité des lipides est la plus faible. Ces variations de la digestibilité apparente des lipides ne semblent pas liées à la teneur des rations en matières grasses. Faut-il en conclure que la nature même des lipides des tourteaux de soja peut être influencée par les traitements thermiques?

Extractif non azoté: On ne note aucune différence appréciable dans les digestibilités moyennes.

Phosphore: La digestibilité du phosphore de la ration 3 est significati- 
vement plus faible que celle des régimes $\mathbf{I}, 2,4$ et 5 (soja 3 et $\mathrm{I}, t=5,98$ soja 3 et $2, t=2,74-\operatorname{soja} 3$ et $4, t=4,75$, soja 3 et $5, t=4,73$ ). Or, le soja 3 est, de tous les tourteaux que nous avons essayés, celui qui apporte le moins de Phosphore $(0,685$ p. IOo). D'un autre côté, on pourra remarquer que la digestibilité du phosphore de la ration à base d'aliment commercial est la plus élevée que nous ayons enregistrée au cours de ces essais. Cette différence peut s'expliquer vraisemblablement par la nature même $d u$ phosphore de ce dernier régime en raison $\mathrm{du}$ fait que, d'une part, l'aliment commercial dont nous sommes partis est un aliment équilibré et que, d'autre part, la proportion d'orge que nous avons utilisée dans le mélange est relativement faible :35 p. roo au lieu de 65-70 p. Ioo pour les autres rations.

Calcium : Les digestibilités moyennes du calcium sont toutes très faibles ; certaines sont même nulles ou négatives. Pour les seuls sojas 3 et 4 , la digestibilité moyenne est positive et toutes les digestibilités individuelles le sont également; elles sont néanmoins très faibles. Aussi peut-on conclure pratiquement que l'apport de calcium de la ration correspond sensiblement aux besoins d'entretien des animaux et que l'influence du traitement thermique des sojas sur l'utilisation digestive du calcium est nulle.

On ne remarque, ni pour l'utilisation digestive du phosphore, ni pour celle du calcium, aucune différence individuelle très nette.

Bilans azotés: L'étude des bilans azotés moyens ( $c f$. tableau $n^{\circ}$ IV), exprimés en $\mathrm{g}$ d'azote par animal et par jour, montre des variations importantes entre les rations expérimentales utilisées. On notera toutefois des différences très larges, particulièrement pour les rations $\mathrm{I}$ et 4 , et c'est ce qui explique que, malgré un écart de plus de II 7 p. Ioo entre les résultats obtenus avec les régimes I et 5 (séries I et IV), la différence entre les bilans azotés moyens ne soit pas statistiquement significative au "point 5 p. Ioo" $(t=2,34)$.

\section{TaBleaU IV}

Bilans azotés

(en g d'azote par animal et par jour)

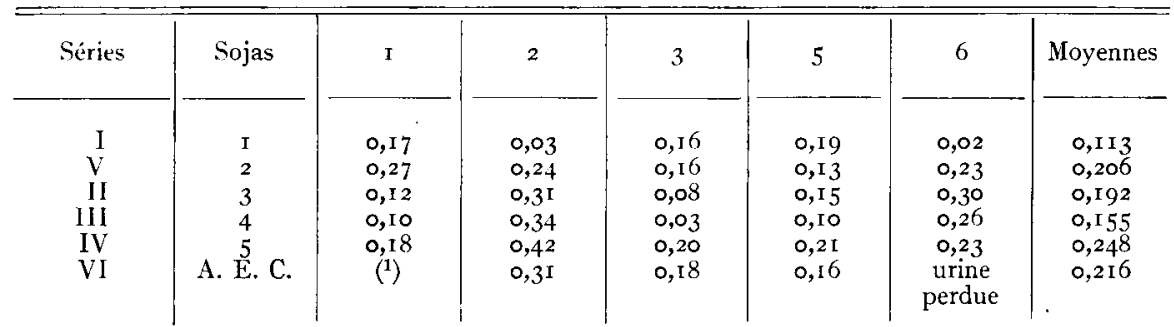

(1) Dans les tableaux IV, $V$ et VI, les chiffres concernant le rat $\mathrm{n}^{0} \mathbf{1}$ font défaut, cet animal étant mort le lendemain de la fin de cette série expérimentale et son poids n'ayant cessé de décroître, nous n'avons pas tenu compte des résultats qu'il avait fournis. 
Il n'en reste pas moins que la mesure du bilan azoté permet de mettre en évidence d'une façon pratique, des écarts notables entre les régimes que nous avons utilisés. Comme la seule variable que nous avons introduite dans la composition de ces régimes est la nature du tourteau de soja qu'ils renferment, nous sommes en droit de conclure que la technique du bilan azoté se révèle assez fine pour permettre un classement des "valeurs en azote " des suppléments protéiques utilisés.

Coefficient de rétention azotée : Cependant, en raison même des différences que nous avons signalées plus haut dans l'utilisation digestive de l'azote des rations expérimentales, il nous paraît que la véritable mesure du rendement de l'azote alimentaire est donnée par la quantité de métalloïde fixée à partir de la quantité qui a traversé la barrière intestinale. Le coefficient de rétention azotée exprime ce "rendement "; on peut. en effet, le calculer à partir du rapport ci-après :

$$
\frac{\mathrm{N} \text { ingéré }-\mathrm{N} \text { fécal }-\mathrm{N} \text { urinaire }}{\mathrm{N} \text { ingéré }-\mathrm{N} \text { fécal }}=\frac{\mathrm{N} \text { du bilan }}{\mathrm{N} \text { absorbé }}
$$

On obtient ainsi les valeurs consignées dans le tableau $\mathrm{V}$.

'TABLEAU V

Coefficient de rétention azotée

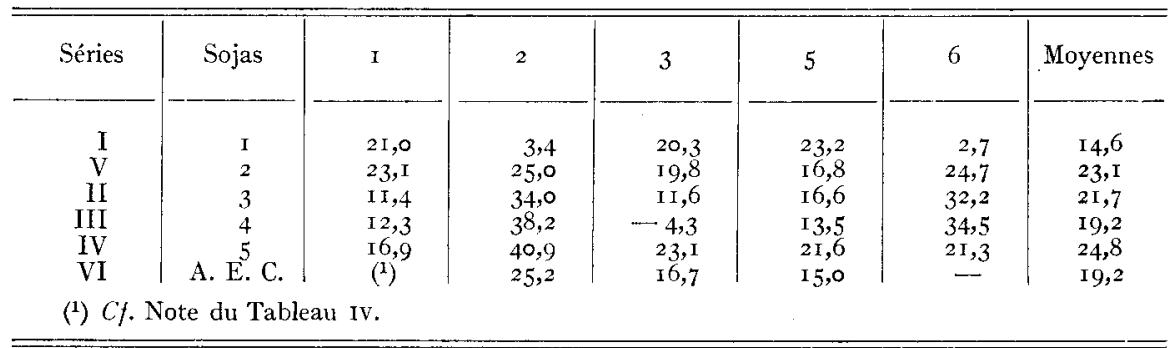

Il se trouve que les résultats obtenus se classent dans le même ordre que ceux fournis par les bilans azotés, au moins en ce qui concerne les rations à base de tourteau de soja. Mais on remarquera que le coefficient de rétention azotée relatif à l'aliment commercial (AEC) fait classer cet aliment en $5^{\mathbf{e}}$ place, alors que le bilan le mettait en seconde place. Nous estimons en tout état de cause, que la " cote " fournie par le coefficient de rétention paraît préférable à celle donnée par le bilan azoté.

\section{Discussion}

Le tableau $n^{\circ}$ VI indique, d'une part le poids moyen des animaux pendant la durée d'expérience, ou moyenne arithmétique de $n$ poids journaliers ( $n$ étant la durée de l'expérience en jours), et d'autre part la variation moyenne quotidienne de poids, c'est-à-dire la différence moyenne entre les poids au début et en fin d'expérience. 
TABLEAU VI

Poids moyen des animaux et variation en cours d'expérience

\begin{tabular}{|c|c|c|c|c|c|c|c|}
\hline \multirow{2}{*}{ Séries } & \multirow{2}{*}{ Sojas } & \multicolumn{5}{|c|}{ Numéros des rats } & \multirow{2}{*}{ Moyenne } \\
\hline & & I & 2 & 3 & 5 & 6 & \\
\hline I & I & 478,5 & 423,3 & 392,7 & 392,7 & $3^{64,3}$ & 410,3 \\
\hline $\mathrm{V}$ & 2 & $\begin{array}{r}-0,7 \\
444,3\end{array}$ & $\begin{array}{l}-\mathrm{I}, \mathrm{r} \\
4 \mathrm{I} 8,8\end{array}$ & $\begin{array}{c}\circ \\
395,7\end{array}$ & $\begin{array}{c}0 \\
398,7\end{array}$ & $\begin{array}{r}-2, \mathrm{I} \\
384, \mathrm{I}\end{array}$ & $\begin{array}{l}-0,8 \\
408,3\end{array}$ \\
\hline II & 3 & $\begin{array}{c}0 \\
476,1\end{array}$ & $\begin{array}{c}0 \\
416,9\end{array}$ & $\begin{array}{r}+0,5 \\
391,2\end{array}$ & $\begin{array}{c}0 \\
394,7\end{array}$ & $\begin{array}{r}+1,0 \\
363,2\end{array}$ & $\begin{array}{r}+0,3 \\
408,4\end{array}$ \\
\hline & & $-1,0$ & 0,6 & $+0,6$ & & $-0,5$ & $-0,3$ \\
\hline III & 4 & 474,9 & 417,7 & 390,3 & 391,3 & 372,3 & 409,3 \\
\hline IV & 5 & $\begin{array}{r}+0,5 \\
476,7\end{array}$ & $\begin{array}{c}\circ \\
408,4\end{array}$ & $\stackrel{\circ}{390,8}$ & $\begin{array}{c}0 \\
392,8\end{array}$ & $\begin{array}{l}-0,3 \\
376,7\end{array}$ & $\begin{array}{c}\circ \\
409, \mathrm{I}\end{array}$ \\
\hline & & (1) & $+1,95$ & $+0,9$ & $+1,25$ & $+-2,6$ & $+1,3$ \\
\hline VI & A. E. C. & (1) & $\begin{array}{r}422,0 \\
-0, \mathrm{I}\end{array}$ & $\begin{array}{l}417,0 \\
+0,85\end{array}$ & $\begin{array}{r}409,0 \\
+0,6\end{array}$ & $\begin{array}{c}391,0 \\
0\end{array}$ & $\begin{array}{r}409,8 \\
+0,3\end{array}$ \\
\hline
\end{tabular}

(') Cf. Note du Tableau Iv.

Ces variations moyennes ne dépassent pas $0,7 \mathrm{p}$. Ioo du poids moyen, ce qui montre que les animaux sont pratiquement en équilibre de poids. Si, poussant plus loin l'analyse des résultats, on compare les bilans azotés moyens et les variations moyennes de poids d'un "rat moyen" (tableau VI colonne " moyenne", on voit qu'avec les sojas qui donnent les plus faibles bilans ( $n^{0}$ I, 3 et 4 ), on obtient des variations moyennes de poids négatives ou nulles, tandis qu'avec les meilleurs sojas, donnant les bilans azotés les plus élevés ( $\mathrm{n}^{\circ} 2$ et 5 ), on obtient un gain de poids des animaux tout au long de l'expérience. Cette observation nous paraît intéressante en ce qu'elle confirme l'opinion courante selon laquelle la partie importante d'un tourteau de soja est sa fraction azotée ; nous avons pu montrer, en effet, que ni pour la digestibilité de la matière sèche totale, ni pour les digestibilités de la matière organique, de la matière minérale ou de l'extractif non azoté, il n'existe de différences notables. Mais nous avons signalé des écarts pour les digestibilités des matières azotées, des matières grasses et du phosphore. La sommation de tous ces effets, telle qu'on peut la mesurer par les variations du poids vif, suit étroitement les variations des caractéristiques du métabolisme de l'azote. Il en résulte, au moins en première approximation, qu'on peut se contenter de n'effectuer que les analyses relatives aux déterminations des teneurs en azote de l'alimentation, des fèces et des urines lorsque, utilisant le rat adulte comme animal-réactif, on cherche à établir la valeur alimentaire relative d'un tourteau de soja. Le travail analytique se trouverait ainsi singulièrement allégé sans que, pour autant, soit affectée la précision des résultats. 


\section{Conclusions}

I. - Les différences que nous avons pu mettre en évidence entre les rations préparées à l'aide de 5 tourteaux de soja diversement traités montrent que la seule détermination de la teneur en azote des ingesta et des excreta permet une classification de la valeur alimentaire de ces tourteaux pour le rat adulte; le bilan azoté ou, mieux, le coefficient de rétention azotée, sert à établir cette classification.

II. — C'est ainsi que, par ordre de valeur alimentaire décroissante, les tourteaux utilisés se classent de la manière suivante : $\mathrm{n}^{\mathrm{o}} 5-\mathrm{n}^{\mathrm{o}} 2-\mathrm{n}^{\mathrm{o}} 3-$ $\mathbf{n}^{0} 4-\mathbf{n}^{0}$ I. Les deux premiers ont des valeurs sensiblement comparables; il en est de même pour les tourteaux $n^{\circ} 3$ et $n^{\circ} 4$; si bien qu'au total, on peut dire que nous avons eu affaire à trois groupes de tourteaux $n^{\circ} 5$ et $2-n^{\circ} 3$ et $4-\mathrm{n}^{\circ} \mathrm{I}$, ce dernier se montrant de loin le plus " mauvais ». Un aliment équilibré du commerce se classe dans le second groupe.

III. - L'étude que nous avons entreprise permet d'indiquer, en outre, l'existence de différences notables dans l'utilisation digestive des matières grasses et du phosphore des rations expérimentales. Mais nous ne sommes pas en mesure de pouvoir attribuer ces différences, soit au traitement thermique des tourteaux, soit à leurs teneurs en ces éléments.

IV. - Une durée de l'ordre de 2 semaines suffit pour obtenir des résultats homogènes, étant entendu que les animaux ont été préalablement habitués à leurs rations pendant une semaine environ.

\section{B. - Eiude de la croissance}

\section{a) Protocole expérimental}

Comme pour l'étude des digestibilités, 6 séries d'expériences ont été réalisées avec les 5 tourteaux de soja et l'aliment complet pour rats. I,es rations furent établies avec la même orge que précédemment, complétée par le même composé minéral, de façon que le régime brut ait une teneur en matières azotées voisine de 26 à $29 \mathrm{p}$. Ioo.

Le schéma des expériences est indiqué dans le tableau no VII.

Les animaux étaient mis en expérience dès leur livraison, c'est-à-dire au sevrage, alors qu'ils étaient âgés de 3 semaines à un mois. Ils étaient pesés 6 fois par semaine. Comme pour les rats adultes, des échantillons de nourriture furent prélevés chaque jour, afin de constituer un échantillon global dont on trouvera les résultats d'analyse dans le tableau no VIII. 


\section{TABleaU VII}

Schéma des expériences de croissance

\begin{tabular}{|c|c|c|c|c|c|c|}
\hline \multirow{2}{*}{ No des sojas } & \multirow{2}{*}{$\begin{array}{c}\text { Durée des } \\
\text { expériences } \\
\text { j }\end{array}$} & \multirow{2}{*}{$\begin{array}{l}\text { Age moyen en } \\
\text { début d'exp. } \\
\underset{j}{ }\end{array}$} & \multicolumn{2}{|c|}{$\begin{array}{l}\text { Nombre de mâles } \\
\text { expér. }\end{array}$} & \multicolumn{2}{|c|}{$\begin{array}{l}\text { Nombre de femelles } \\
\text { expér. }\end{array}$} \\
\hline & & & début & fin & début & fin \\
\hline I & 45 & $2 \mathrm{I}$ & Io & 6 & IO & IO \\
\hline 2 & 45 & $2 \mathrm{I}$ & Io & 8 & IO & 8 \\
\hline 3 & 43 & 27 & 8 & 8 & 9 & 8 \\
\hline 4 & 45 & 22 & IO & 9 & 8 & 6 \\
\hline 5 & 45 & 22 & I3 & I3 & Io & IO \\
\hline A. E. C. & 43 & 27 & 7 & 6 & IO & I0 \\
\hline
\end{tabular}

TABLEAU VIII

Composition des rations ( $g$ pour $100 \mathrm{~g} \mathrm{sec)}$

\begin{tabular}{|c|c|c|c|c|c|c|c|c|}
\hline Série & Composition des rations $\%$ & M. M. & M. C. & M. A. & M. G. & E. N.A. & $\mathrm{P}$ & $\mathrm{Ca}$ \\
\hline $\mathrm{I}$ & $\begin{array}{ll}\text { Orge } & 55 \% \\
\text { Soja I } & 45\end{array}$ & $6,3^{1}$ & 5,55 & 29,66 & $3,5^{8}$ & 54,90 & 0,63 & $0.6 \mathrm{I}$ \\
\hline II & $\begin{array}{ll}\text { Orge } & 45 \\
\text { Soja z } & 55\end{array}$ & 6,34 & 6,97 & $32,4 \mathrm{I}$ & 3,47 & $50,8 \mathrm{I}$ & 0,68 & $0,6 \mathbf{I}$ \\
\hline III & $\begin{array}{ll}\text { Orge } & 50 \\
\text { Soja } 3 & 50\end{array}$ & 6,02 & 5,57 & $3^{2,20}$ & 2,73 & $53,4^{8}$ & 0,67 & 0,63 \\
\hline $\mathrm{V}$ & $\begin{array}{ll}\text { Orge } & 50 \\
\text { Soja } 4 & 50\end{array}$ & 6,43 & 5,34 & 32,58 & 2,89 & $52,7^{6}$ & 0,66 & 0,66 \\
\hline IV & $\begin{array}{ll}\text { Orge } & 50 \\
\text { Soja } 5 & 50\end{array}$ & 6,47 & 5,96 & 32,60 & 2,75 & 52,22 & 0,67 & $0,7 I$ \\
\hline $\mathrm{VI}$ & $\begin{array}{l}\text { Orge } \\
\text { A. E. C. } 100\end{array}$ & 8,19 & 4,26 & 28,27 & 5,02 & 54,26 & $\mathrm{I}, \mathrm{IO}$ & 1,70 \\
\hline
\end{tabular}

\section{Tableau IX}

Poids moyens et gains moyens de poids vit.

\begin{tabular}{|c|c|c|c|c|c|c|c|}
\hline \multirow{2}{*}{ No des sojas } & \multicolumn{2}{|c|}{$\begin{array}{l}\text { Poids moyen des } \\
\text { mâles en } \mathrm{g}\end{array}$} & \multicolumn{2}{|c|}{$\begin{array}{l}\text { Poids moyen des } \\
\text { femelles eng }\end{array}$} & \multicolumn{3}{|c|}{ Gain moyen en $g$ par jour } \\
\hline & début & fin & début & fin & mâles & femelles & Total \\
\hline I & 42 & 150,5 & 40 & I 4 I & 2,3 & 2,2 & 2,3 \\
\hline 2 & $4 \mathrm{I}$ & 127 & $3^{8}$ & $13^{8}$ & 1,9 & 2,2 & $2, \mathrm{I}$ \\
\hline 3 & 42 & 172 & 43 & 147 & 3,0 & 2,4 & 2.7 \\
\hline 4 & 38 & 144 & $3^{8}$ & 130 & 2,4 & 2,0 & 2,2 \\
\hline 5 & 36 & 129 & 39 & 127 & 1,9 & 2,0 & 2,0 \\
\hline A. E. C. & 36 & $18 \mathrm{I}$ & $4 \mathrm{I}$ & 148 & 3,3 & 2,5 & 2,9 \\
\hline
\end{tabular}




\section{b) Résultats}

L es seules mesures qui ont été effectuées sont les pesées des animaux. Des essais furent entrepris pour peser les ingesta, mais ils n'aboutirent pas. Les

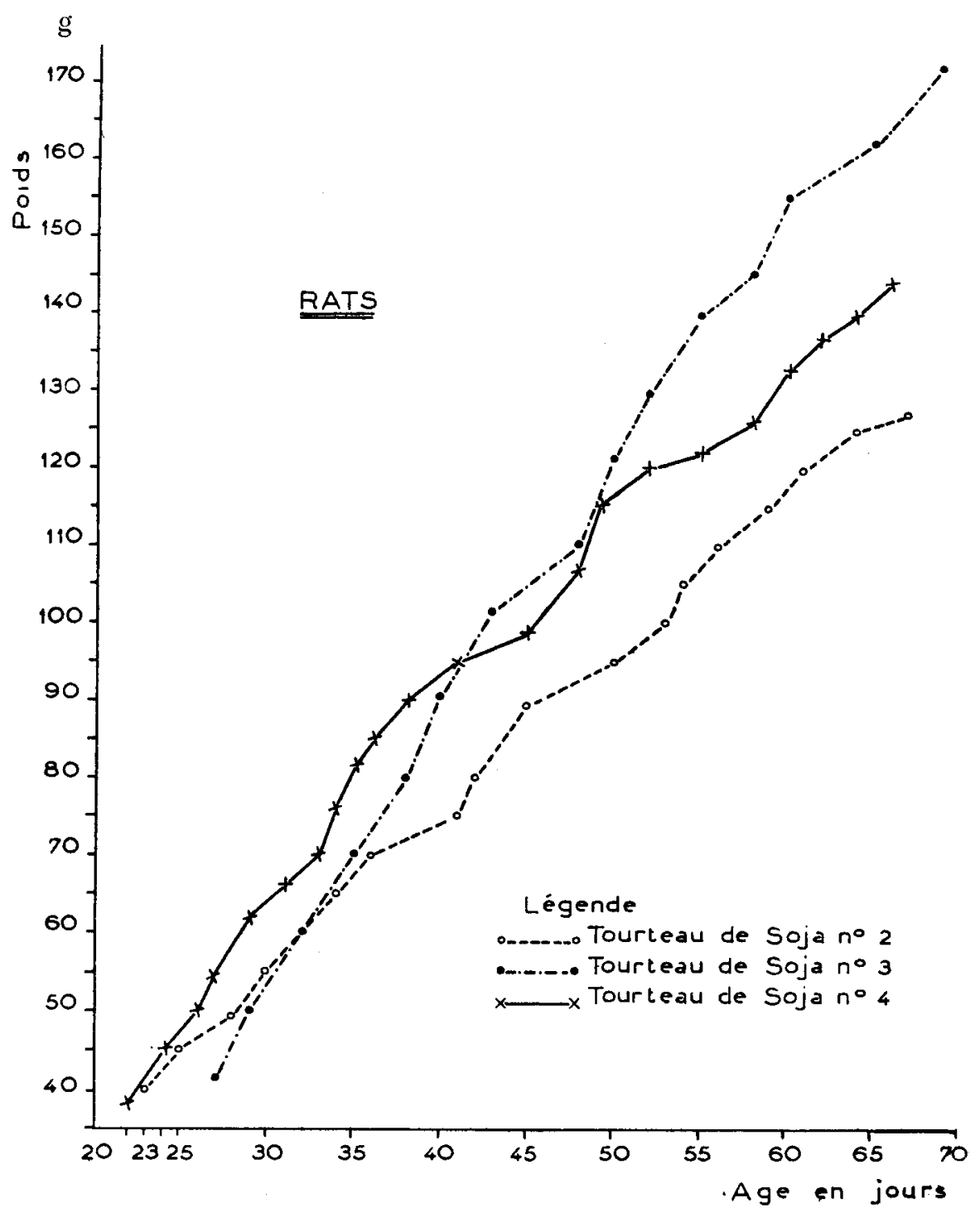

FIG. 2

cages où les animaux étaient placés sont des cages pour rats de réserve et ne sont pas conçues pour séparer l'urine et les fèces des refus de nourriture ; d'autre part, les déchets de nourriture sont éparpillés dans toute la cage et non 
pas seulement au-dessous des mangeoires. De sorte que la pesée des refus, qui aurait permis la connaissance des ingesta, s'étant révélée impossible, nous n'avons pu calculer le rendement de la nourriture ni l'indice de consommation, et nous avons dû nous contenter de mesurer la croissance pondérale des animaux.

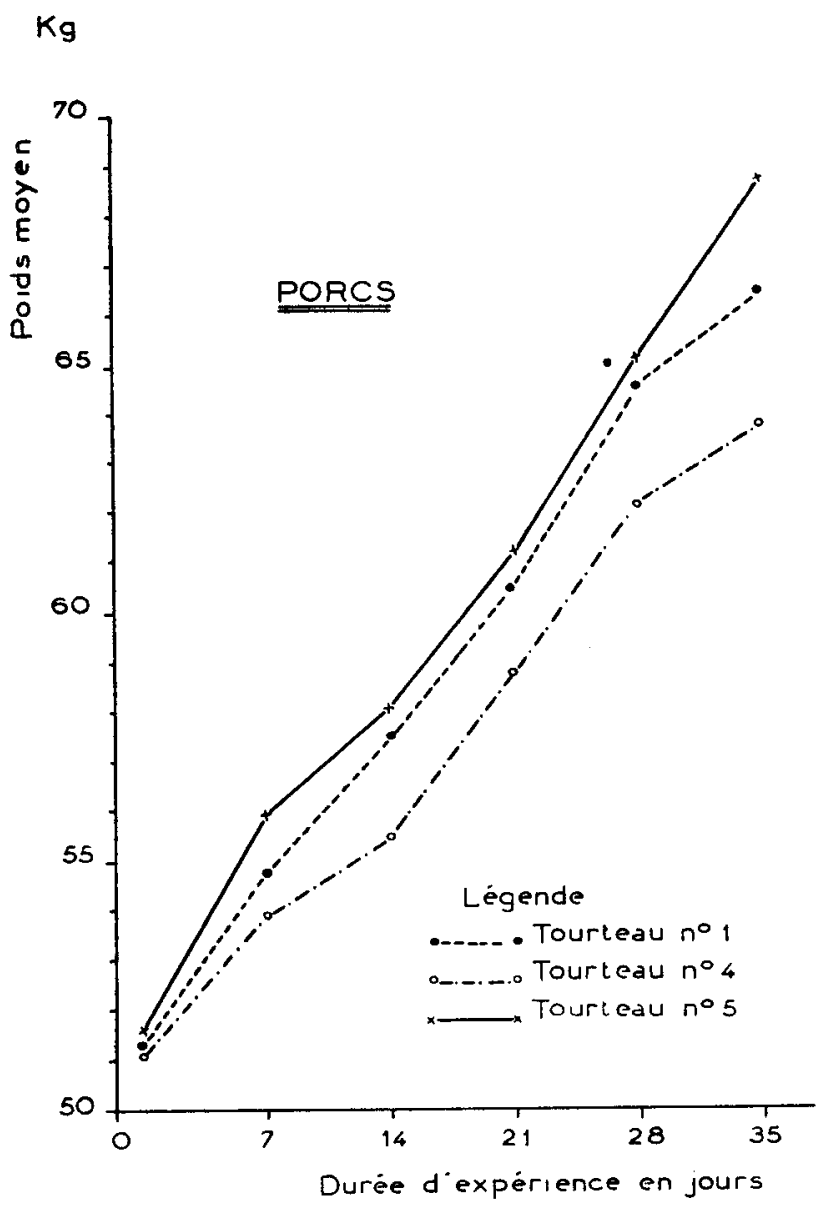

FIG. 3.

Le tableau IX indique les poids moyens au début et à la fin des expériences et les gains moyens par jour. On voit qu'à part l'aliment commercial qui fournit les meilleurs résultats, c'est la ration $\mathrm{n}^{0} 3$ qui assure la croissance la plus rapide pour les mâles comme pour les femelles; au contraire, les rations $n^{0} 2$ et 5 pour les mâles et $n^{\circ} 4$ et 5 pour les femelles, ne permettent qu'un développement relativement lent des animaux. 
D'autre part, les différences relatives entre les gains moyens extrêmes sont nettement plus élevées chez les mâles que chez les femelles.

$$
\begin{aligned}
& \text { Mâles : Rations 2, } 5 \text { et } 3 \quad \frac{3,0-\mathbf{I}, 9}{\frac{3,0+1,9}{2}}=\frac{2,2}{4,9}=45 \% \\
& \text { Femelles : Rations 4, } 5 \text { et } 3 \quad \frac{\frac{2,4-2,0}{2,4+2,0}}{2}=\frac{0,8}{4,4}=18 \% .
\end{aligned}
$$

Cette constatation est aussi très nette quand on étudie les courbes de croissance (fig. 2). Ceci prouve que les femelles sont moins sensibles que les mâles aux variations de régime et accusent moins qu'eux les améliorations ou les déficiences d'une ration, en raison vraisemblablement de ce que leurs besoins sont moindres.

\section{Conclusions}

I. - Ainsi, en comparant les gains moyens de poids par jour pour un " rat moyen ", on obtient, en plaçant les tourteaux par ordre de valeur alimentaire décroissant, le classement suivant : en tête, le tourteau $n^{\circ} 3$; pratiquement équivalents, les tourteaux $n^{\circ} 4$ et $I$; enfin les tourteaux no 2 et 5 .

II. - Pour de semblables mesures, il est indiqué de n'utiliser que des rats mâles, les réactions de ceux-ci étant nettement plus marquées que celles des femelles.

\section{CONCLUSIONS GÉNÉRALES}

Au terme de cette étude, il s'agit de savoir si l'on peut valablement utiliser le rat pour éprouver la valeur alimentaire d'un tourteau de soja destiné au porc.

I. - Pour cela, reprenant les conclusions des mesures réalisées sur porcs, nous voyons que l'on peut classer les cinq tourteaux, d'après leur rendement pour la croissance - indice de consommation - dans l'ordre suivant, du meilleur au moins bon : 3, 2, 5, I et 4 . Le tourteau $n^{0} 3$ vient nettement en tête du classement, les quatre autres pouvant être groupés par deux ( 2 et 5 -- I et 4), d'efficacité voisine.

Si l'on rapproche ces conclusions de celles que nous a fournies l'étude de la croissance du rat, on constate ici encore que le tourteau $\mathrm{n}^{0} 3$ se classe en tête. Mais par contre, si les quatre autres tourteaux se trouvent également groupés par deux ( I et $4-2$ et 5), l'ordre de classement est inverse de celui trouvé pour le porc.

Que conclure de ces faits expérimentaux ? A notre sens, il semble possible d'affirmer que la seule mesure de la croissance sur rat ne peut donner de résul- 
tats certains applicables directement au porc que lorsque le tourteau de soja a subi un traitement améliorant nettement sa valeur alimentaire. I,orsque les tourteaux ont des valeurs alimentaires assez proches les unes des autres, soit en raison de leur nature même, soit en raison d'un traitement thermique inadéquat, la méthode de la croissance du rat ne permet pas d'établir une classification rigoureuse valable pour le porc.

II. - La composition des tourteaux en acides aminés est peut-être capable d'expliquer ces divergences. Si l'on se reporte aux tableaux $\mathrm{X}_{1}$ et $\mathrm{X}_{2}$ du mémoire, on voit que le tourteau $n^{0} 3$ se sépare nettement des autres par ses teneurs élevées en cystine et en méthionine : o,89 p. Ioo et o,6o p. Ioo respectivement. Si l'on tente de classer les 5 tourteaux essayés par ordre de teneur décroissante en cystine + méthionine, on arrive aur résultat suivant: 3-I-2-4-5.

Mis à part le tourteau no 3 , ce classement ne correspond à aucun de ceux que nous a donnés la double expérience biologique.

D'autre part, les teneurs en tryptophane sont assez différentes, comprises entre 0,79 et $\mathrm{r}$, II $\mathrm{p}$. Ioo et ne permettent pas non plus un classement rendant compte de l'une ou de l'autre mesure de croissance.

Ces observations nous conduisent à penser que,étant donnéela composition très spéciale de nos rations, c'est la teneur en cystine et en méthionine du régime qui, à partir d'un certain seuil, conditionne le rendement de la croissance. En dessous de ce seuil - qui paraît se placer chez le rat aux environs de 0,45 p. Ioo pour la cystine et de $0,30 \mathrm{p}$. Ioo pour la méthionine $\left(0, \mathrm{I}_{4} \mathrm{p}\right.$. Ioo et 0,09 p. roo chez le porc) - d'autres facteurs interviennent qui masquent plus ou moins l'influence des acides aminés soufrés; et parmi ces facteurs, l'un de nous a pu mettre en évidence l'appétence de la ration offerte aux animaux et la nature même des graines de soja.

III. - Chauffage des tourteaux. - Comme on aura pu le remarquer, nous ne possédons pas de données très précises sur les conditions du traitement thermique qu'avaient subi les tourteaux : tantôt nous ignorons la température de séchage après extraction, tantôt la durée de l'exposition à une température donnée. Pour pallier ce manque de renseignements, nous avons effectué sur tous les tourteaux l'épreuve à l'uréase (5). D'après ce test, il est malheureusement impossible de savoir si un tourteau a été suffisamment chauffé ou au contraire surchauffé, puisque, dans les deux cas, 1'uréase est détruite, alors que ces deux traitements ont une action très différente sur la valeur nutritive des matières azotées.

Quoi qu'il en soit, on peut aboutir au classement suivant, par ordre d'intensité décroissante du traitement thermique (variation croissante du $\mathrm{pH}$ ) : 3 et 2 -I et 5-4. Cet ordre est très voisin de celui qui a été trouvé dans l'expérience réalisée sur porcs $(3,2,5, \mathbf{I}, 4)$. Faut-il en conclure que cette espèce animale est plus sensible que le rat aux variations de structure ou de composition que la chaleur peut provoquer dans les tourteaux? 
IV. - Enfin, notre étude montre qu'on ne peut en aucune façon utiliser la méthode des bilans azotés sur rat adulte lorsqu'il s'agit d'apprécier la valeur alimentaire d'un tourteau de soja pour animaux en croissance. Ce résultat n'a rien qui puisse surprendre : on sait bien que les besoins qualitatifs de l'entretien ne sont pas les mêmes que ceux de la croissance; il est regrettable qu'une méthode aussi rapide nécessitant peu de matériel et peu d'animaux, se revèle inutilisable dans le cas présent. Mais cette étude a eu du moins l'avantage de mettre en évidence le fait que le traitement thermique des sojas influence, non seulement l'utilisation digestive de la fraction azotée, mais aussi celle des matières grasses et de certains composants de la fraction minérale.

Concluons donc que la mesure de la croissance du rat est capable de donner des résultats entièrement superposables à ceux obtenus sur porcelets lorsque le tourteau à étudier, ayant subi un traitement thermique adéquat, présente une valeur alimentaire élevée. Elle ne fournit que des données approximatives dans le cas où le traitement appliqué n'a pas provoqué une amélioration nette de la valeur alimentaire. Toutefois, les erreurs de classification auxquelles on pourrait être ainsi amené ne paraissent pas devoir présenter des conséquences pratiques très importantes: les différences mises en évidence par les deux méthođes biologiques utilisées sont, en effet, à la limite même de la précision des techniques employées dans chaque cas. Il s'ensuit que, en raison de la simplicité de l'expérimentation sur rat en croissance et de son prix de revient peu élevé, les résultats que fournit ce mode d'appréciation de la valeur alimentaire des tourteaux de soja nous paraissent pouvoir être appliqués [sans grands risques à l'espèce porcine.

\section{BIBLIOGRAPHIE}

I) FÉvrter (R.). - L'indice de consommation est-il le témoin fidèle de l'efficacité d'une ration? Annales de Zootechnie ; 1 ; I, 1952.

(2) Jacquot (R.), Matet (J.) et Fridenson (O.). - Influence de traitements thermiques et industriels sur la valeur protidique des aliments. Annales de la Nutrition et de l'Alimentation ; 1 ; 2 ; I 57 , 1947 .

(3) Leroy (A. M.) et Février (R.). - Influence du mode de distribution des repas sur l'utilisation de la nourriture par le porc. Annales Agronomiques ; 5 ; 769 , 1947.

(4) Leroy (A. M.) et FÉvrier (R.). - Nombre des repas et utilisation de la nourriture par le porc. Annales Agronomiques; 4 ; 605, 1949 .

(5) Urease activity and the chemical criteria as indications of inadequate heating of Soybean Oil Meal. J. of A.O.A.C. $30 ; 354$, I947. 